\title{
Three Cases of Bilateral Breast Absence Associated with Familial Congenital Ectodermal Defects
}

This article was published in the following Dove Press journal: Clinical, Cosmetic and Investigational Dermatology

\section{Shunqing Su \\ Rurong Xie \\ Xiumei Ding \\ Yuechun Lin}

Department of Burn \& Plastic Surgery, Dalang Hospital of Dongguan, Dongguan, 523770, People's Republic of China
Correspondence: Shunqing Su Department of Burn \& Plastic Surgery, Dalang Hospital of Dongguan, No. 85 of Jinlang Middle Street, Dalang Town, Dongguan, 523770, People's Republic of China

Tel +8676983009512

Fax +8676983009171

Email sushunqing_dr88@163.com
Objective: This study aims to analyze three cases of bilateral breast absence associated with congenital ectodermal defects in the same family to identify a suitable clinical treatment plan.

Methods: Three patients (case 1 and case 2 are a brother-sister relationship; case 3 is their father) complained of the absence of breasts, nipples, and areolas, accompanied by deformity of facial features and fingers; all other clinical indexes were normal. Case 1 first underwent bilateral papillary reconstruction, with areola embroidery carried out six months later. Case 2 first underwent prosthetic breast augmentation, and after ten months, she underwent nipple reconstruction and auricular cartilage, silica gel prosthesis rhinoplasty, epicanthus correction, and areola embroidery. Gene tests were carried out for both cases. Case 3 did not undergo any surgical procedures.

Results: The operations achieved good results, although in case 2, the reconstructed nipples retracted and became smaller. Neither of the subjects had adverse reactions after the procedures. A heterozygous mutation of the KCTD1 gene c.2020A>T (p.i674f), a mutation inherited from case 3 (their father), was detected through gene analysis. Copy number analysis and single-nucleotide polymorphism (SNP) analysis were carried out, but no copy number variation possibly related to clinical manifestations was detected.

Conclusion: The bilateral breast absence associated with familial congenital ectodermal defects in cases 1 and 2 were found to be induced by a heterozygous mutation of the KCTD1 gene c.2020A $>\mathrm{T}$ (p.i674f) inherited from case 3 (their father). Two of the three cases underwent surgical treatment, and good clinical results were achieved.

Keywords: bilateral breast absence associated with congenital ectodermal defects, familial inheritance, breast reconstruction, nipple reconstruction, case report

\section{Introduction}

Congenital breast absence is a rare congenital malformation. Its genetic model is unclear, and its clinical manifestations are greatly varied. ${ }^{1}$ It was first reported in 1839 by Froriep, who proposed that the condition was caused by the development stagnation of the mammary ridge in the sixth week of embryo development. $^{2}$ Some drugs may also affect embryonic development, leading to congenital breast absence. ${ }^{3}$

The condition is characterized by the absence of breasts and nipples, unilaterally or bilaterally. It can also have multiple clinical manifestations. For example, it is often accompanied by one or more deformities, such as congenital 
eye, ear, and nose deformities, craniomaxillofacial deformities, and limb deformities. ${ }^{1}$ This kind of physiological defect is also associated with psychological pressure and social disorders. ${ }^{1}$ Breast and nipple reconstructions are usually needed to improve the psychological outcomes for the patients. ${ }^{4}$

There are many surgical breast reconstruction procedures. The most commonly used procedures include prosthetic breast reconstruction, prosthesis reconstruction after tissue expansion, and reconstruction of the breast using the back's broadest muscle flap and various types of abdominal rectus muscle flaps. ${ }^{5}$ However, each procedure has advantages and disadvantages. As such, doctors should comprehensively consider the patient's condition and situation when trying to make appropriate treatment choices. ${ }^{6}$

In this study, three bilateral breast absence cases associated with congenital ectodermal defects in the same family were analyzed. Two of the three cases underwent surgical treatment. The details and outcomes of these cases can provide a suitable clinical treatment plan for this condition.

\section{Research Methods \\ Clinical Data}

Case 1: The patient (the brother of case 2) was a 17-yearold male admitted to the hospital with a lifelong absence of bilateral breasts and nipples.

Physical examination: temperature $(\mathrm{T}): 36.5^{\circ} \mathrm{C}$; pulse $(\mathrm{P})$ : 68 beats/minute; respiration (R): 20 breaths/minute; blood pressure (BP): 108/67 mmHg. The shape of the head was normal, the distance between the eyes was increased, single eyelid epicanthus (Figure 1A and C). No abnormality was found in the heart and lungs, the aperture of the thorax had no malformation, and bilateral nipple areolas were absent (Figure 2A). No gland tissue was palpated. Bilateral axillary hair was sparse, and no axillary web was found (Figure 2B). The fingers of both hands were short, the distal phalanx of the little fingers presented with slight flexion and deformity, and both hands were webbed (Figure 1B). Chest magnetic resonance imaging (MRI): bilateral greater and lesser pectoral muscles were normal, and no malformation was found in the ribs. Breast color Doppler ultrasound: no glandular echo was found in the subcutaneous space of the bilateral
A

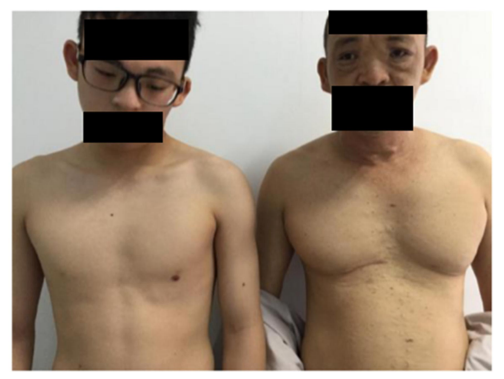

B

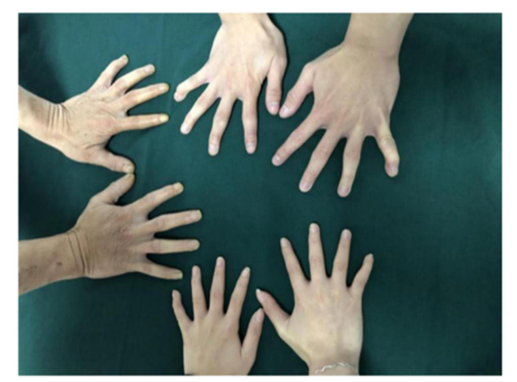

C

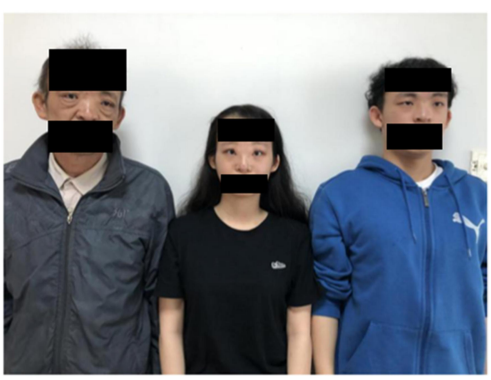

Figure I (A) Preoperative facial and chest signs of Case I and Case 3. (B) Hand deformity of Case I, Case 2, Case 3. Upper: the father; below: the sister; left: the brother. (C) Facial characteristics of Case I, Case 2 (postoperative) and Case 3.

A

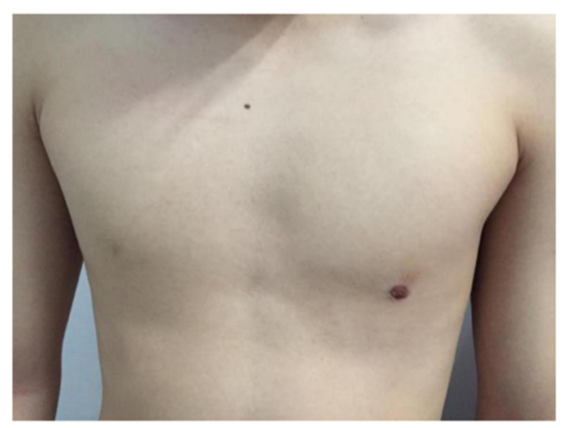

B

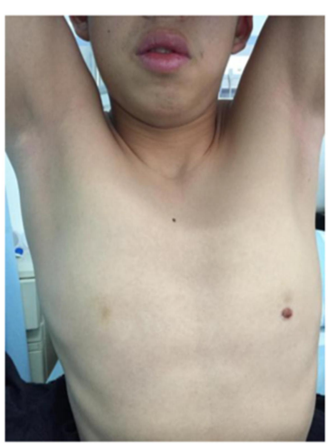

C

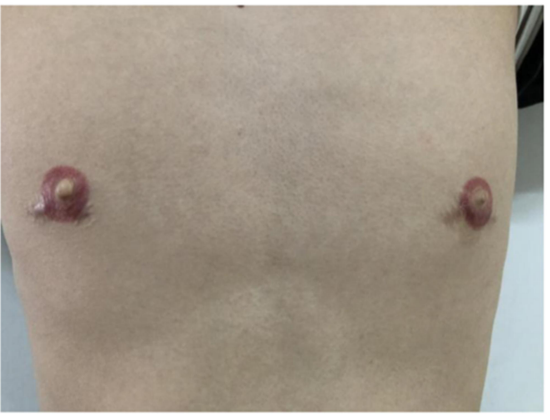

Figure 2 Preoperative and postoperative chest signs of Case I. (A) Preoperative chest signs of Case I (the lower left is artificial skin injury, not nipple). (B) Sign of axillary hair absence in Case I. (C) Chest sign of Case I one year after nipple reconstruction and embroidery. 
chest walls. Color Doppler ultrasound of the liver, gallbladder, spleen, and urinary system: no abnormalities were found.

Case 2: The patient (the sister of case 1) was a 22-yearold female admitted to the hospital with a lifelong bilateral planar chest and absence of nipples.

Physical examination: T: $36.3^{\circ} \mathrm{C}$; P: 104 beats/minute; R: 20 breaths/minute; BP: 131/81 mmHg. The shape of the head was normal, the oculi rimae was small, the orbital distance was significantly widened, double eyelids presented with epicanthus, the subcutaneous fat layer between eyebrows was thick, the bridge of the nose was low and flat, and bilateral ears were cup-shaped (Figure 3A and B). The hands were not webbed, the fingers of both hands were short, and the distal phalanx of the little fingers presented with slight flexion and deformity (Figure 1B). Tooth enamel was absent, and the arrangement of teeth was irregular. No abnormality was found in the heart and lungs, the aperture of the thorax was not malformed, bilateral nipple areolas were absent, and the chest was planar (Figure 3A). No gland tissue was palpated. No axillary hair or sweat secretion was found under the armpits, and pubic hair was sparse. Chest MRI: bilateral greater and lesser pectoral muscles were normal. No deformity was found in the ribs. Breast color Doppler ultrasound: no glandular echo was found in the subcutaneous space of the bilateral chest walls. Color Doppler
A

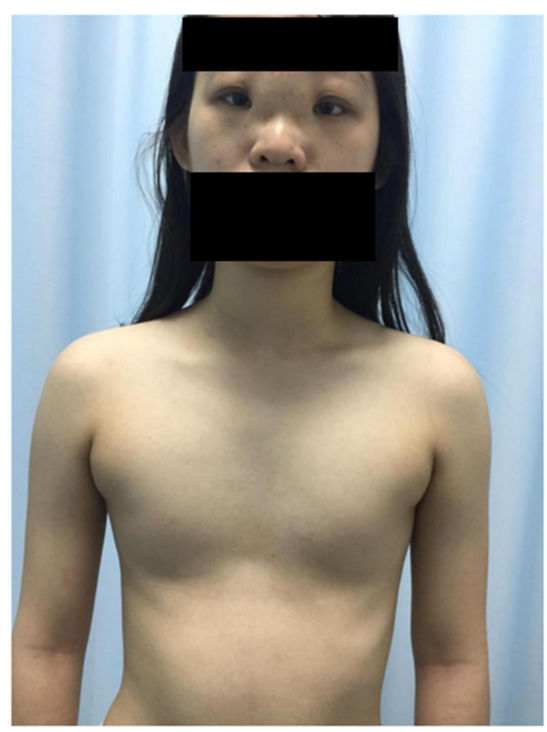

C

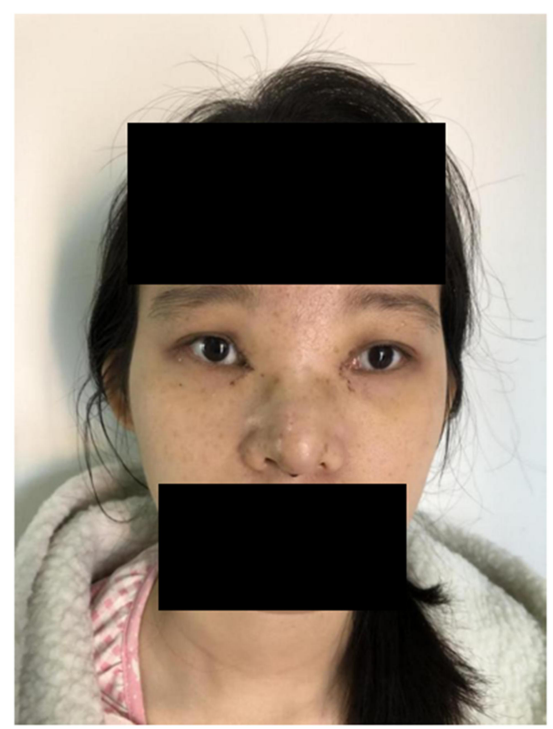

B

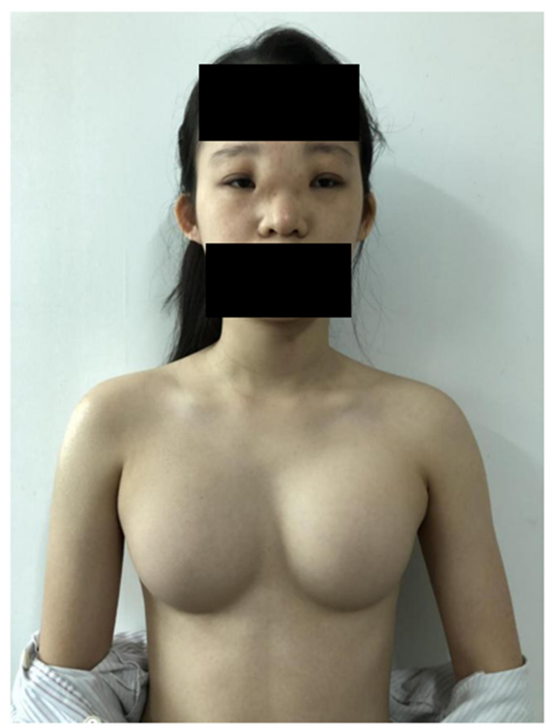

D

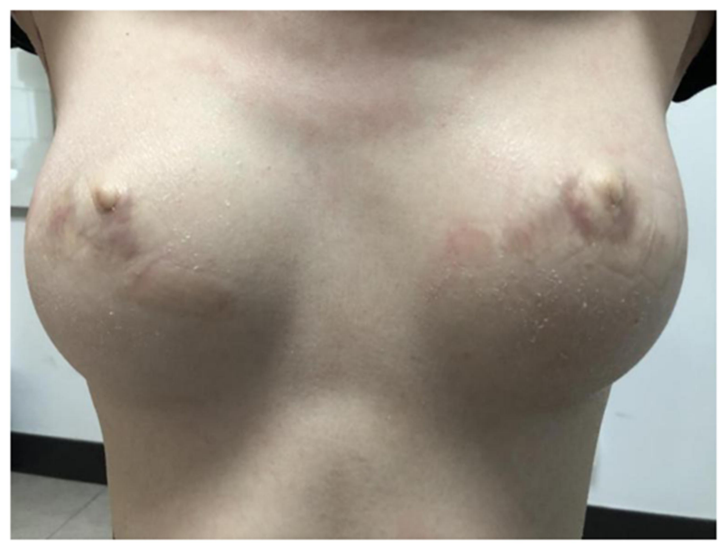

Figure 3 Preoperative and postoperative facial and chest signs of Case 2. (A) Preoperative facial and chest signs of Case 2. (B) Chest sign one year after breast reconstruction. (C) Facial signs one month after eye and nose plastic surgery. (D) Chest sign one year after breast reconstruction. 
ultrasound of the liver, gallbladder, spleen, and urinary system: no abnormalities were found.

Case 3: The patient (the father of cases 1 and 2) was a 50-year-old male who was not admitted to the hospital.

Physical examination: The shape of the head was normal, the orbital distance was significantly widened, the nose presented with saddle malformation, and the ears were protruding (Figure 1A and $\mathrm{C}$ ). The aperture of the thorax was malformed, greater pectoral muscles were hypertrophic, and bilateral nipple areolas were absent (Figure 1A). No gland tissue was palpated. Bilateral axillary hair was sparse, and no axillary web was found. The fingers of both hands were short, the distal phalanx of the little fingers presented with slight flexion and deformity, and the hands were webbed (Figure 1B). Breast color Doppler ultrasound: no glandular echo was found in the subcutaneous space of the bilateral chest walls.

The study was conducted in accordance with the Declaration of Helsinki (as was revised in 2013). The study was approved by Ethics Committee of the Dalang Hospital of Dongguan (NO.DLYY20200216). All patients provided informed consent for the case details and images to be published in the study.

\section{Surgical Methods}

Case 1 first underwent bilateral papillary reconstruction, with areola embroidery carried out six months later. A follow-up was conducted three years after the operations (Figure 2C).

Case 2 underwent first-stage prosthetic breast augmentation with $200 \mathrm{~mL}$ of silica gel prosthesis. After ten months, she underwent nipple reconstruction and auricular cartilage, silica gel prosthesis rhinoplasty, and epicanthus correction (Figure 3C). One year after nipple reconstruction, her areolas were embroidered. During follow-ups, the shapes of her breasts and areolas were good (Figure 3D).

Case 3 did not undergo surgery.

For patients with congenital unilateral breast absence, the positioning of the inferior wrinkle on the affected side can be determined based on comparison with the opposite side. However, for patients with congenital bilateral breast absence, where there is no defined position of the inferior wrinkle to act as a guide, it is difficult to locate the reconstructed breast. Patients with congenital breast absence do not experience the feeling of breasts and are physically not ready to decide where they would like their breasts. In this study, the method introduced by Lin was followed: ${ }^{1}$ before the operation, the patient was asked to wear a bra with a prosthetic model and to adjust the bra to a comfortable position; this position also met the aesthetic criteria of the doctors. The lower edge of the bra was marked to determine the inferior wrinkle of the breast; this line was shifted down by $1-2 \mathrm{~cm}$ when stripping the lacuna. Female nipple reconstruction for case 2 was carried out one year after breast reconstruction, as the skin had good elasticity at that time, and the donor area can be directly sutured without tension. The nipple was located at the highest point of the breast. The male nipple was located by finding the intersection of the vertical line at the middle point of the clavicle. This location was $17-21 \mathrm{~cm}$ from the sternocleidomastoid line. The patient was also consulted, and appropriate adjustments were made. In summary, it is crucial to identify the most appropriate timing and surgical method for reconstruction of the breasts and nipples based on the patient's situation.

Based on a patient's situation and requirements, their facial features may also need to be corrected. In this study, auricular cartilage and silica gel prosthesis rhinoplasty and correction of the epicanthus were performed for case 2, and good clinical effects were achieved.

\section{Results}

\section{Follow-Up Results After the Operations}

Prostheses were implanted into the greater pectoral muscles of case 2, which were fully covered by tissue. A follow-up was conducted one year later, during which the breasts felt good to the touch, and it was concluded that the surgery had achieved excellent results.

Bilobate flaps were used to form the nipples in both cases 1 and 2. The areolas were embroidered six months to one year after the first operation, and the shapes were lifelike. However, in case 2, the reconstructed nipples retracted and became smaller. No adverse reactions were found after the procedures in either of the cases.

Case 2 also underwent facial correction, including auricular cartilage and silica gel prosthesis rhinoplasty, and correction of the epicanthus. Good clinical effects were achieved, and no adverse reactions were identified after the operation.

\section{Chromosome Examination Results}

High-precision clinical single-exon person detection and analysis were carried out on the genes of cases 1 and 2, detecting a heterozygous mutation of the KCTD1 gene c.2020A $>$ T (p.I674F). This mutation can also be named 
c.196A>T (p.I66F) (transcript: NM_001258221). Later validation experiments revealed that the mutation was inherited from case 3, their father (heterozygous state). Copy number analysis and single-nucleotide polymorphism (SNP) analysis were also carried out, but no copy number variation possibly related to clinical manifestations was detected ${ }^{7}$ (Table 1).

\section{Discussion}

Congenital breast absences are generally classified into three categories according to their clinical manifestations: bilateral breast absence associated with congenital ectodermal defects, congenital unilateral breast absence, and congenital bilateral breast absence. ${ }^{4}$ Abnormal development of skin and its accessory organs (hair, exocrine glands, and sebaceous glands), teeth, and nails is usually characterized by sparse body hair, saddle-nose deformity, and wide ocular distance. Female patients have deformities of the teeth and ears but no other musculoskeletal defects. In the present study, three patients complained of the absence of breasts or nipple areolas, and all had facial and finger deformities. Therefore, the patients in this study can be diagnosed with bilateral breast absence associated with congenital ectodermal defects.

The genetic pattern of bilateral breast absence associated with congenital ectodermal defects is a sex chromosome recessive inheritance, which means it has a $50 \%$ chance of being inherited by a son from his mother. Pedigree analysis of pure species revealed that the genetic pattern in females is autosomal or sex chromosome dominant inheritance. ${ }^{5}$ However, in the present study, the condition was inherited by the children from their father. No similar conditions were found in their other parent or grandparents; the family was not a consanguineous marriage family. The study detected a heterozygous mutation of the KCTD1 gene c.2020A $>$ T (p.I674F), which can also be named c.196A $>$ T (p.I66F) (transcript: NM_001258221). Validation experiments demonstrated that the mutation was inherited from the father (heterozygous state). This new missense mutation has not been reported in related clinical cases, and it has not been reported in our reference population gene database either. The region of mutation is an important part of this protein, and the amino acid sequences of different species are highly conserved. The computer-aided analysis predicted that this mutation is more likely to affect protein structure/function. In summary, based on the clinical manifestations and family

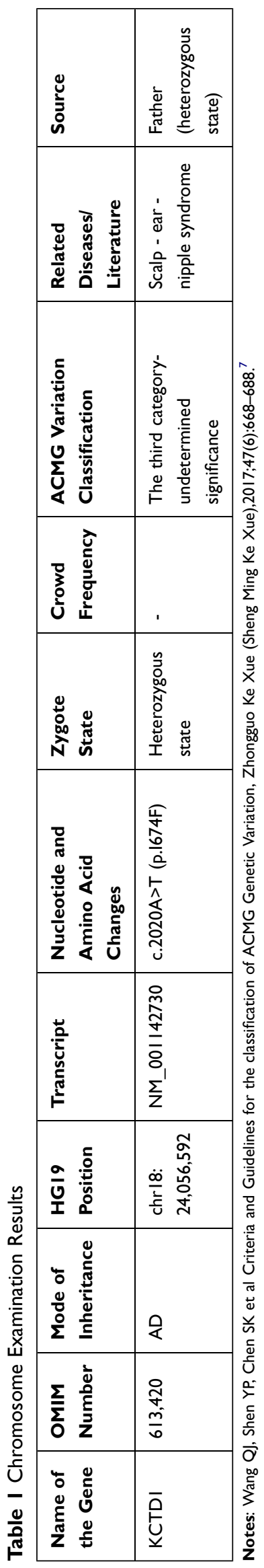


analysis of the patients, this mutation can be categorized in the American College of Medical Genetics and Genomics (ACMG) mutation classification guidelines (PMID: 25,741,868) as "the third categoryundetermined significance." KCTD1 gene-related diseases are scalp-ear-nipple syndromes, which have an autosomal dominant inheritance pattern. The main clinical manifestations are scalp cuticle hypoplasia, slight abnormality of the external ears, and breast deformity; some patients also have nail dystrophy, abnormal teeth, fingers, and toes, and kidney deformity, although there are significant differences in individual manifestations (PMID: 23,541,344).

Patients with an absence of breasts or nipple areolas need breast and nipple reconstruction. There are many surgical breast reconstruction procedures. The most commonly used procedures include prosthetic breast reconstruction, prosthesis reconstruction after tissue expansion, and reconstruction of the breast with the back's broadest muscle flap and various types of abdominal rectus muscle flaps. ${ }^{6}$ Compared with acquired breast absence, congenital breast absence is usually only accompanied by a deficiency of tissue quantity, not skin defects; therefore, prosthetic breast or breast reconstructions after tissue expansion are often adopted. ${ }^{8}$ In the present study, the female patient had relatively good skin elasticity on her chest and complete greater pectoral muscles. There was sufficient tissue for coverage after the prosthesis was implanted into the greater pectoral muscles. A follow-up was conducted one year later, during which the reconstructed breasts felt good, and it was concluded that the operation had achieved excellent results.

There are many possible procedures for reconstructing the nipple complex, such as with autogenous tissue (for example, the earlobe, auricle, cartilage transplantation, or toe pulp transplantation and reconstruction), flap reconstruction, reconstruction of dermal scar tissue, and tissue substitute fillings. Flap reconstruction can provide more tissue and results in good blood circulation and recovery; it also allows the nipple to be relatively straight and maintain a certain height. However, this method usually needs to be performed 6-12 months after breast reconstruction. In this study, bilobate flaps were used to form nipples in both cases 1 and 2. Six months to a year later, the areolas were embroidered with lifelike shapes. ${ }^{9}$ However, in case 2 , the reconstructed nipples retracted and became smaller, which was also noted in related reports. $^{10}$

\section{Conclusion}

Three cases of bilateral breast absence associated with familial congenital ectodermal defects were analyzed. It was found that the condition was inherited from the father (case 3), but the cause of the genetic defect is unknown. Two of the three patients underwent surgical treatment, and good clinical effects were achieved. The study showed that it is vital to identify the most appropriate timing and surgical method for reconstructing the breasts and nipples based on the patient's situation.

\section{Acknowledgments}

We are particularly grateful to all the people who have given us help on our article.

\section{Funding}

There is no funding to report.

\section{Disclosure}

The authors declare that they have no competing interests.

\section{References}

1. Lin KY, Nguyen DB, Williams RM. Complete breast absence revisited. Plast Reconstr Surg. 2000;106(1):98-101. doi:10.1097/ 00006534-200007000-00018

2. Tiainen S, Masarwah A, Oikari S, et al. Tumor microenvironment and breast cancer survival: combined effects of breast fat, M2 macrophages and hyaluronan create a dismal prognosis. Breast Cancer Res Treat. 2020;179(3):565-575. doi:10.1007/s10549-019-05491-7

3. Rajan R, Tunkel DE. Choanal atresia and other neonatal nasal anomalies. Clin Perinatol. 2018;45(4):751-767. doi:10.1016/j. clp.2018.07.011

4. Reyes-Reali J, Mendoza-Ramos MI, Garrido-Guerrero E, MéndezCatalá CF, Méndez-Cruz AR, Pozo-Molina G. Hypohidrotic ectodermal dysplasia: clinical and molecular review. Int J Dermatol. 2018;57 (8):965-972. doi:10.1111/ijd.14048

5. Ritwik P, Patterson KK. Diagnosis of tooth agenesis in childhood and risk for neoplasms in adulthood. Ochsner J. 2018;18(4):345-350. doi:10.31486/toj.18.0060

6. Knackstedt R, Deross A, Moreira A. Congenital breast deformities: anatomical basis and surgical approaches for optimal aesthetic outcomes. Breast J. 2020;26(7):1302-1307. doi:10.1111/tbj.13798

7. Wang QJ, Shen YP, Chen SK, et al. Criteria and guidelines for the classification of ACMG genetic variation. Zhongguo Ke Xue. 2017;47 (6):668-688.

8. Lee J, Park HS, Lee H, et al. Post-operative complications and nipple necrosis rates between conventional and robotic nipple-sparing mastectomy. Front Oncol. 2021;10:594388. doi:10.3389/ fonc. 2020.594388

9. Feng S, Zhang B, Kappos EA, Tremp M, Yang C. Modified S-flap for nipple reconstruction. Aesthetic Plast Surg. 2017;41(2):312-317. doi:10.1007/s00266-017-0789-z

10. Dong J, Wang T, Zhang Y, Xu H, He J. Staged immediate nipple reconstruction with tube flap in secondary autologous breast reconstruction. J Plast Surg Hand Surg. 2019;53(4):204-207. doi:10.1080/2000656X.2019.1582423 


\section{Publish your work in this journal}

Clinical, Cosmetic and Investigational Dermatology is an international, peer-reviewed, open access, online journal that focuses on the latest clinical and experimental research in all aspects of skin disease and cosmetic interventions. This journal is indexed on CAS.
The manuscript management system is completely online and includes a very quick and fair peer-review system, which is all easy to use. Visit http://www.dovepress.com/testimonials.php to read real quotes from published authors.

Submit your manuscript here: https://www.dovepress.com/clinical-cosmetic-and-investigational-dermatology-journal 